\title{
Étude du comportement mécanique d'un polyamide 66 chargé de fibres de verre courtes
}

\author{
Ghorbel Atidel ${ }^{1,2, a}$, Nicolas Saintier ${ }^{2}$, Abderrazak Dhiab $^{1}$ Et Fakhreddine Dammak ${ }^{1}$ \\ 1 Unité de mécanique modélisation et production (U2MP), École Nationale d'ingénieurs de Sfax, BPW 3038 Sfax, Tunisie \\ 2 Arts et Métiers ParisTech (centre de Bordeaux-Talence), I2M-DUMAS, UMR 5295, Esplanade des Arts et Métiers, \\ 33405 Talence Cedex, France
}

Reçu le 8 août 2009, accepté le 9 mai 2011

\begin{abstract}
Résumé - Cette étude expérimentale présente l'étude du comportement mécanique d'un polyamide 66 (PA66) renforcé par des fibres de verre courtes fréquemment utilisées dans l'industrie automobile. Afin d'étudier l'influence de la teneur en humidité, de la quantité de fibres de verre introduite dans la matrice et de la vitesse de déplacement, une série d'essais de traction a été réalisée sur du polyamide renforcé ou non avec des fibres de verre courtes à différents fractions volumiques : 10, 20 et $30 \mathrm{wt} \%$. Les résultats montrent la dépendance du matériau aux différents paramètres cités précédemment. La technique de thermographie infrarouge (IR) a été utilisée pour déterminer l'évolution de la température à la surface des matériaux au cours des essais de traction. Un processus de localisation de la dissipation thermique a pu être identifié à partir des images thermiques.
\end{abstract}

Mots clés : Polyamide 66 / fibres de verres / rupture / thermographie infrarouge

\begin{abstract}
Study of the mechanical behaviour of a short glass fiber-reinforced polyamide 6,6. In this experimental work, we studied the mechanical behaviour of a short glass fibre reinforced polyamide frequently used in the automotive industry. In order to investigate the influence of glass fibre content, relative humidity and strain rate, we carried out uniaxial tensile tests on an unfilled polyamide and glass fibre reinforced polyamide with different weight fractions: 10, 20 and $30 \mathrm{wt} \%$. Experimental results showed that the studied composite is a strain rate, moisture and fibre volume fraction dependant mechanical behaviour. The thermography infrared (IR) technique has been used to determine the change of the surface temperature at the surface of materials during tensile tests. The local deformation process can be identified and studied from the infrared thermal images.
\end{abstract}

Key words: Polyamide 6,6 / glass fibers / damage / infrared thermography (IR)

\section{Introduction}

Les besoins de l'industrie dans le domaine des matériaux doivent satisfaire à des exigences de plus en plus sévères et souvent antagonistes : facilité et rapidité de mise en œuvre, coût modéré, caractéristiques mécaniques élevées, résistance aux agressions chimiques et thermiques, aptitude au recyclage, etc. Ceci a conduit au développement spectaculaire d'un certain nombre de matériaux parmi lesquels on trouve les composites à matrice thermoplastique et fibres courtes. Différentes technologies permettent la mise en ouvre de ces composites mais c'est le moulage par injection qui est le procédé le

${ }^{a}$ Auteur pour correspondance : gh_atidel@yahoo.fr plus largement utilisé. Le moulage par injection, outre le fait qu'il soit parfaitement adapté à la production en grande série, permet la réalisation, en une seule étape, des pièces complexes pouvant intégrer de multiples fonctions. Cet avantage est particulièrement bien mis à profit dans l'industrie automobile.

Ces dernières années, le marché des thermoplastiques renforcés de fibres courtes est dominé par les polyamides et par les polypropylènes, les fibres de renfort étant des fibres de verres dans la grande majorité des cas. Le polyamide $66\left(\mathrm{PA}_{66}\right)$ est l'un des polymères thermoplastiques les plus utilisés dans l'industrie mécanique, en particulier dans l'automobile et l'aéronautique. Il présente de bonnes propriétés mécaniques, une bonne résistance à la fatigue et à l'usure, un faible coefficient de frottement, un bon 
comportement à la chaleur et au froid et à certains produits chimiques [1].

Le fait de renforcer le $\mathrm{PA}_{66}$ par ajout de fibres de verre courtes permet de lui apporter un certain nombre de qualités supplémentaires tout en conservant l'essentiel de ses qualités initiales. Parmi les apports positifs liés au renforcement, on peut noter la plus grande stabilité dimensionnelle des pièces injectées due à la limitation des retraits et post-retraits ainsi que l'augmentation des caractéristiques mécaniques. Les propriétés mécaniques de ce type de composite ont été le sujet de plusieurs études : elles résultent de la combinaison des propriétés de la matrice, des fibres de verre et de l'interface matrice/fibre de verre. Elles dépendent également des conditions d'injection (vitesse et pression d'injection, température du moule, etc.) [2-4] et des conditions de sollicitation (vitesse de déformation, température d'essai, etc.) [5,6]. L'effet d'autres variables à savoir le diamètre, la longueur, l'orientation et la quantité des fibres de verre a été étudié $[6,7]$.

En raison de leur structure chimique, certains polymères sont sensibles à l'humidité : c'est le cas du polyamide 66. L'eau a pour effet de supprimer la liaison d'hydrogène entre les groupes amide, ce qui augmente la mobilité des chaînes de polyamide et provoque ainsi une modification du comportement mécanique [8,9]. Pour cela, différentes conditions d'hygrométrie ont été choisies (humidité relative : 50, 60 ou $70 \%$ ).

On se propose ici de conduire une étude visant à l'analyse expérimentale, de l'influence de la teneur en humidité, de la quantité de fibres de verre introduite dans la matrice et de la vitesse de déformation sur le comportement mécanique et le mécanisme d'endommagement d'un $\mathrm{PA}_{66}$ chargé avec des fibres de verre courtes soumis à une sollicitation de traction uniaxiale. Les techniques de thermographie infrarouge et de microscopie électronique à balayage ont été utilisées afin d'identifier les mécanismes d'endommagement.

\section{Investigation expérimentale}

\subsection{Matériaux et conditions d'essai}

Les éprouvettes haltères type IA (norme NF EN ISO 527-2) testées lors de cette étude ont été obtenues par moulage injection. Des essais préalables nous ont permis de sélectionner une gamme de paramètres d'injection standard utilisables avec tous les matériaux qu'ils soient ou non renforcés de fibres (vitesse d'injection : $30 \mathrm{~mm} . \mathrm{s}^{-1}$; pression d'injection : 100 bar; temps de refroidissement : $60 \mathrm{~s}$ ). Les granulés ont été préalablement séchés à $80{ }^{\circ} \mathrm{C}$ avant d'être injectés dans le moule.

Dans le cadre de notre étude les paramètres étudiés sont le taux de fibres (0 wt \%, $10 \mathrm{wt} \%, 20 \mathrm{wt} \%$ et $30 \mathrm{wt} \%)$, la vitesse de déformation $\left(5,5 \times 10^{-4} \mathrm{~s}^{-1}, 5,5 \times 10^{-3} \mathrm{~s}^{-1}\right.$ et $\left.5,5 \times 10^{-2} \mathrm{~s}^{-1}\right)$, et le taux d'hygrométrie $(50 \mathrm{RH}, 60 \mathrm{RH}$ et $70 \mathrm{RH})$. Tous les matériaux à différents taux de fibre ont été testés aux différentes vitesses de déformation et taux d'hygrométrie.

\subsection{Moyens d'essai}

Les essais de traction ont été réalisés à température ambiante sur une machine ZWICK/Roell Z250 de capacité $250 \mathrm{kN}$ équipée d'un système de fixation des éprouvettes par mors auto-serrant. Cette machine est équipée d'un extensomètre laser modèle LE-01. Des mires réfléchissantes sont placées sur chaque éprouvette afin de mesurer la déformation dans la zone utile de l'éprouvette au cours de l'essai de traction.

Pour observer les éventuelles évolutions du champ de température à la surface de l'éprouvette accompagnant le processus de déformation, un dispositif de thermographie infrarouge a été associé aux moyens d'essais de traction. Ce dispositif est constitué d'une caméra infrarouge Cedip Jade III à matrice de détecteurs InSb à plan focal de $320 \times 240$ pixels, travaillant dans le domaine des longueurs d'ondes de 3 à $5 \mu \mathrm{m}$ (avec une précision de $0,02{ }^{\circ} \mathrm{C}$ à $20{ }^{\circ} \mathrm{C}$ ). Cette caméra a été utilisée à une fréquence d'acquisition de $50 \mathrm{~Hz}$ (full-frame). L'objectif consiste à associer aux phénomènes de déformation et d'endommagement des phénomènes thermiques locaux (champ de température). Du fait de l'hétérogénéité importante des matériaux de l'étude et de la nature des processus de déformation mis en jeux (localisations aux petites échelles), les hypothèses classiquement utilisées pour l'identification des sources de dissipation (diffusivité homogène à l'échelle des hétérogénéités du champ thermique, invariance dans la direction normale au plan d'observation) ne peuvent très raisonnablement utilisées. Pour cette raison nous nous contenterons dans le cadre de cet article, d'analyser directement l'évolution des champs thermiques. Il est important de noter que la faible diffusivité thermique de la matrice nous permet plus facilement de rapprocher les échelles de localisation du champ thermique à celles, sous-jacentes, des localisations de déformation ou d'endommagement.

Dans le cas des matériaux chargés, des observations en microscopie optique de la section transverse des éprouvettes ont mis en évidence la présence d'une couche de polymère continue de l'ordre d'une dizaine de microns (voir Fig. 1) à la surface des éprouvettes de telle sorte que les fibres ne débouchent pas à la surface (zone de mesure du champ de température). Ceci nous permet de considérer que l'émissivité de la surface pour les matériaux chargés et non chargés sont identiques.

La microscopie optique et électronique à balayage ont été utilisées afin d'identifier les mécanismes d'amorçage et d'évolution de l'endommagement ainsi que la rupture des matériaux.

\section{Résultats et discussion}

\subsection{Comportement en traction}

\subsubsection{Effet du taux de fibres de verre}

La figure 2 illustre les courbes contrainte-déformation pour chaque taux de charge à une vitesse de déformation 


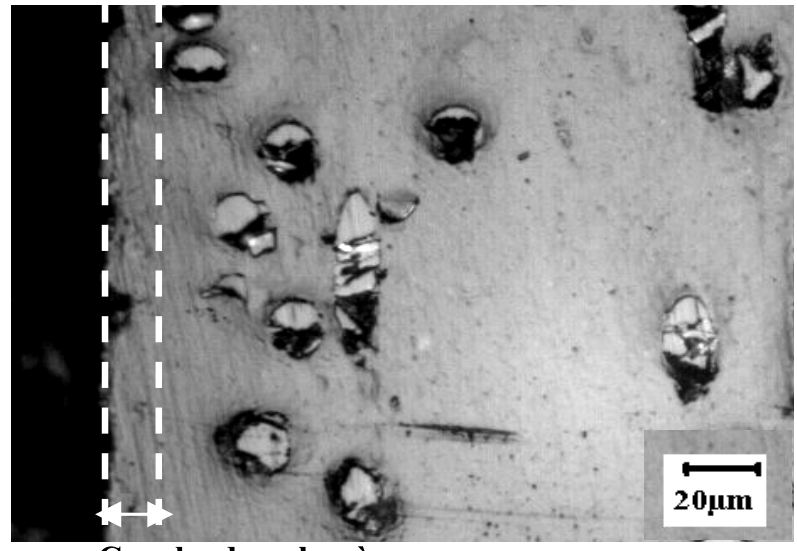

Couche de polymère

Fig. 1. Micrographie optique indiquant une couche superficielle de polymère à la surface.

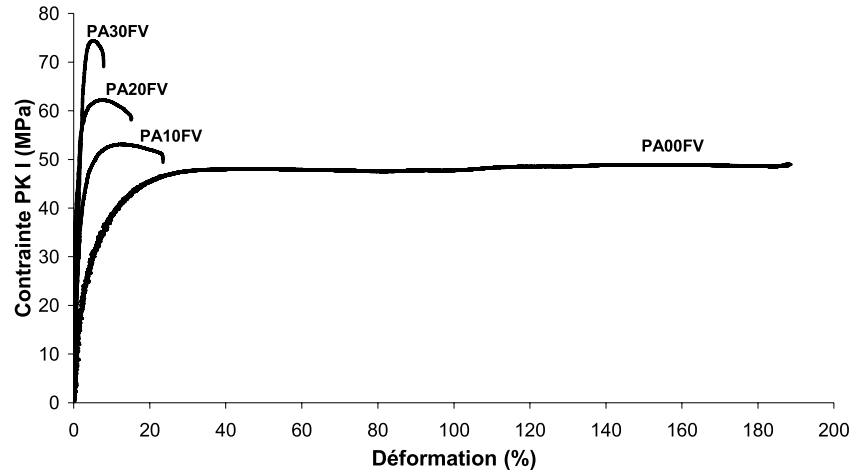

Fig. 2. Courbes contrainte-déformation du $\mathrm{PA}_{66}$ naturel (PA00FV) et chargé à 10 (PA10FV), 20 (PA20FV) et $30 \%$ $(\mathrm{PA} 30 \mathrm{FV})$ à $\dot{\varepsilon}=5,5 \times 10^{-4} \mathrm{~s}^{-1}$ et $\mathrm{RH}=50$.

égale à $5,5 \times 10^{-4} \mathrm{~s}^{-1}$ et un taux d'hygrométrie égal à $50 R H$. Nous avons constaté que l'augmentation du taux de fibres de verre améliore la résistance mécanique du polyamide et diminue sa ductilité. Le même résultat est observable quels que soient le taux d'hygrométrie et la vitesse de déformation.

Pour chaque matériau, les variations des caractéristiques mécaniques (contrainte à rupture, déformation à rupture et module de Young) en fonction du taux de fibres de verres sont indiquées : figures 3, 4 et 5. Des conclusions identiques peuvent être établies : l'augmentation du taux de fibres induit une augmentation de la contrainte à rupture et du module de Young tandis que la déformation à rupture diminue quelle que soit la vitesse de déformation. L'effet de l'ajout des fibres de verres est plus marqué sur la déformation à rupture lors du passage à un taux de $10 \%$ de fibres de verre.

Ces différents résultats sont en accord avec la littérature $[5,10]$.

\subsubsection{Effet de l'augmentation de la vitesse de déformation}

Pour des taux de fibres et d'hygrométrie constant, l'augmentation de la vitesse de déformation a pour effet

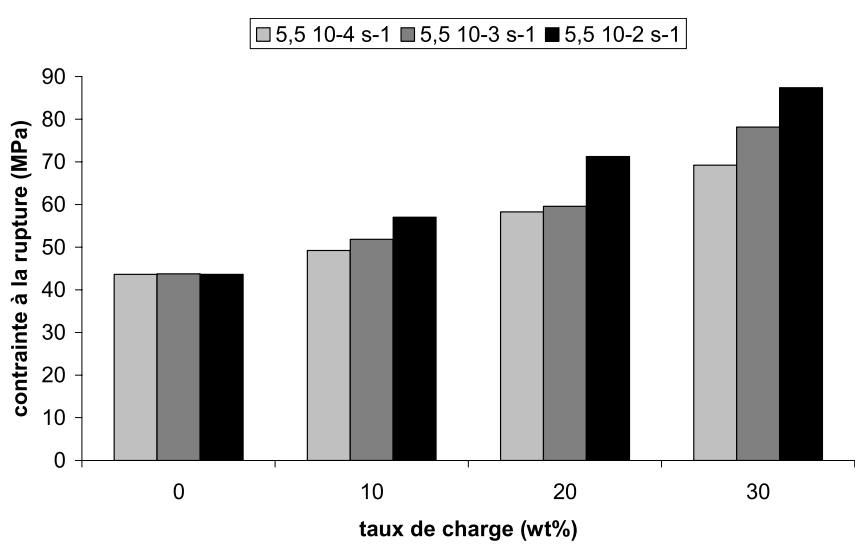

Fig. 3. Variation de contrainte à la rupture en fonction du taux de fibres de verre à $\mathrm{RH}=50$.

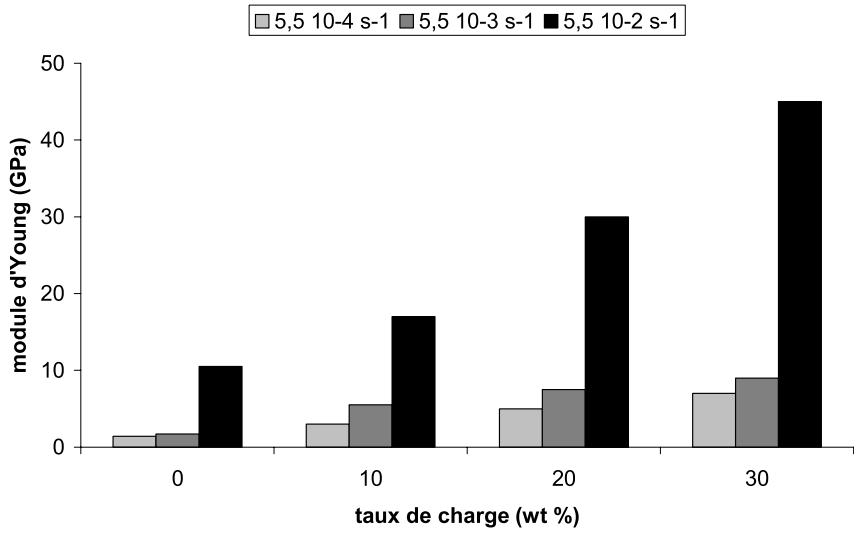

Fig. 4. Variation du module de Young en fonction du taux de fibres de verre à $\mathrm{RH}=50$.

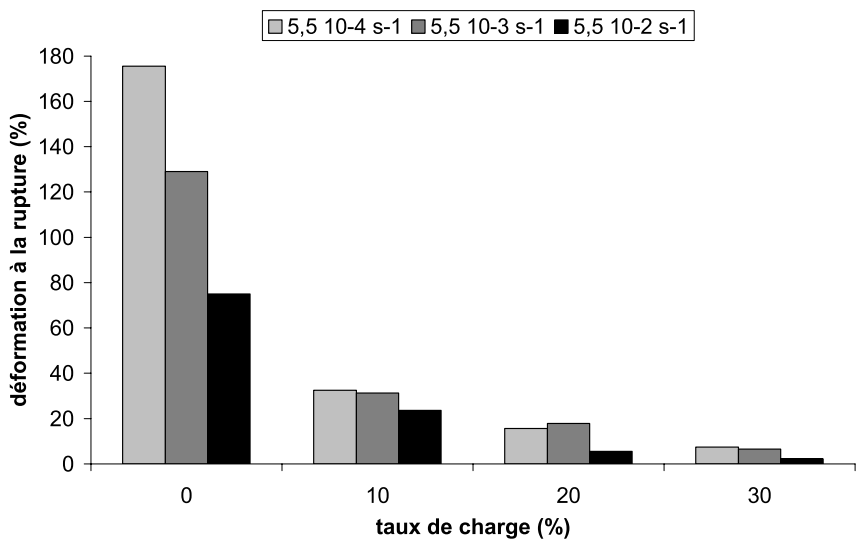

Fig. 5. Variation de déformation à la rupture en fonction du taux de fibres de verre à $\mathrm{RH}=50$.

l'augmentation du module d'élasticité, de la contrainte à la rupture et la diminution de la déformation à la rupture (Figs. 3-5). Cet effet diminue lorsque le taux de fibres diminue.

Dans le cas du polymère non chargé, l'effet de la vitesse de déformation est différent. La figure 5 montre que 


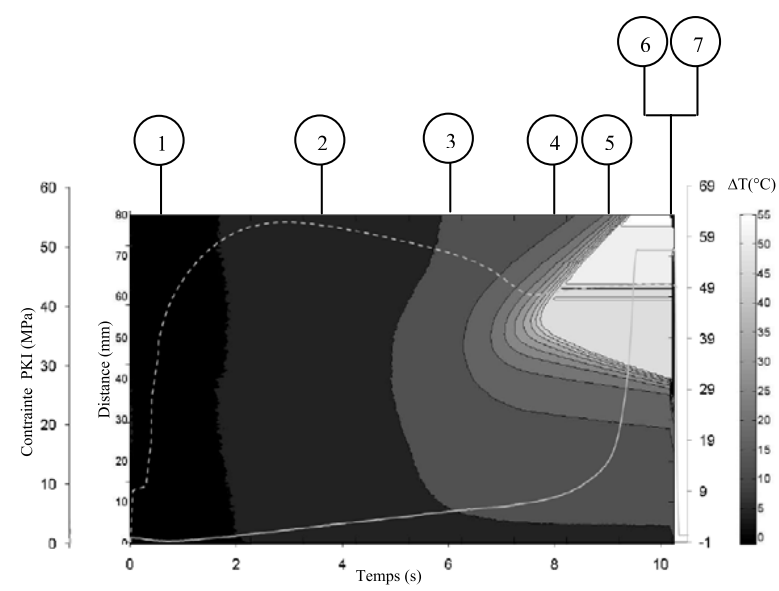

(a)
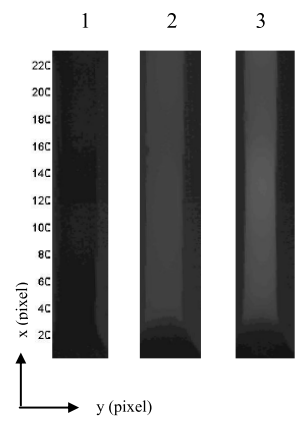

(b)

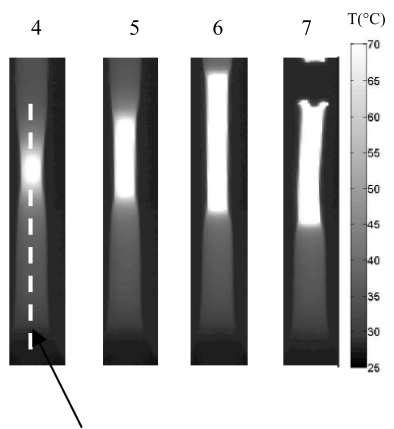

ligne d'analyse

Fig. 6. (a) Courbe 1 : évolution temporelle des profils longitudinaux-courbes 2 et 3 : évolution de la charge et de la température (en un point particulier); (b) photogrammes thermiques de la surface de l'éprouvette à des temps différents montrant l'évolution des lèvres de striction du PA00FV (1 pixel représente $0,5 \mathrm{~mm}$ ).

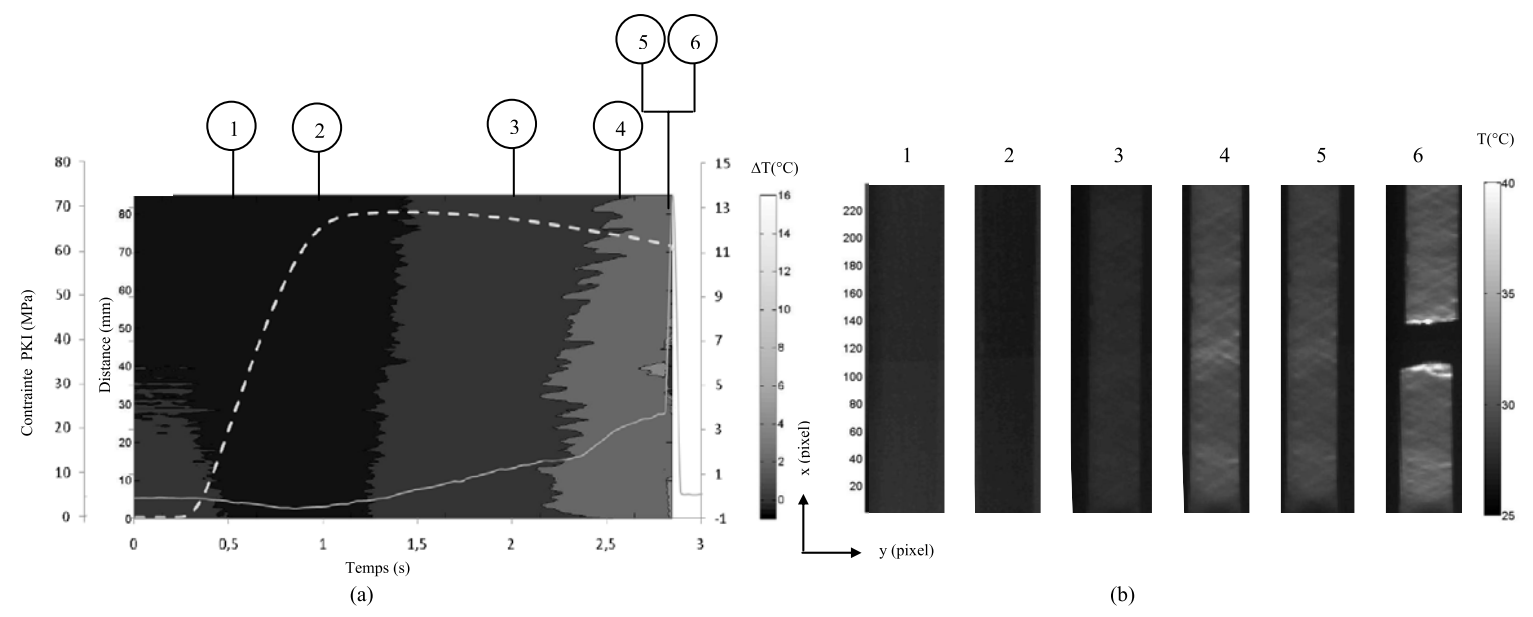

Fig. 7. (a) Courbe 1 : évolution temporelle des profils longitudinaux-courbes 2 et 3 : évolution de la charge et de la température (en un point particulier); (b) photogrammes thermiques de la surface de l'éprouvette à des temps différents du PA10FV (1 pixel représente $0,5 \mathrm{~mm})$.

l'augmentation de la vitesse de déplacement de l'essai entraîne :

- un effet non significatif sur le module d'élasticité tangent jusqu'à la vitesse $5,5 \times 10^{-3} \mathrm{~s}^{-1}$. Au-delà de cette valeur, une augmentation du module de Young est notée;

- une baisse de la déformation à rupture. Cette réduction de ductilité peut être expliquée par le fait que plus la vitesse de déformation est importante, moins les molécules ont le temps de se réarranger [5].

\subsubsection{Effet du taux d'hygrométrie}

Nous avons noté que le comportement en traction du polymère non chargé est affecté par l'augmentation de l'humidité : diminution du module élastique et de la déformation à rupture (l'effet de l'augmentation de l'humidité est non significatif sur le module d'élasticité tangent entre RH 50 et RH 60). Les mêmes tendances ont été constatées dans le cas du polymère chargé. Les différents résultats obtenus sont valables quels que soient le taux de charge et la vitesse de déformation.

\subsection{Analyse thermographique}

Dans cette analyse, seuls les résultats sur matériau traité à RH 50 sont présentés.

Les courbes des figures $6 \mathrm{a}, 7 \mathrm{a}, 9 \mathrm{a}$ et $11 \mathrm{a}$ font correspondre le chargement mécanique et le profil du champ de température le long d'une ligne sur l'axe de l'éprouvette. L'évolution de la température en un point particulier de la matière est aussi tracée. Cette évolution permet d'estimer l'ordre de grandeur des variations de température obtenues pour les différentes conditions d'essais. Ces courbes montrent que la réponse thermique est totalement différente entre le polymère non chargé (PA00FV) et chargé de fibres de verre courtes (PA10FV, PA20FV et PA30FV). 


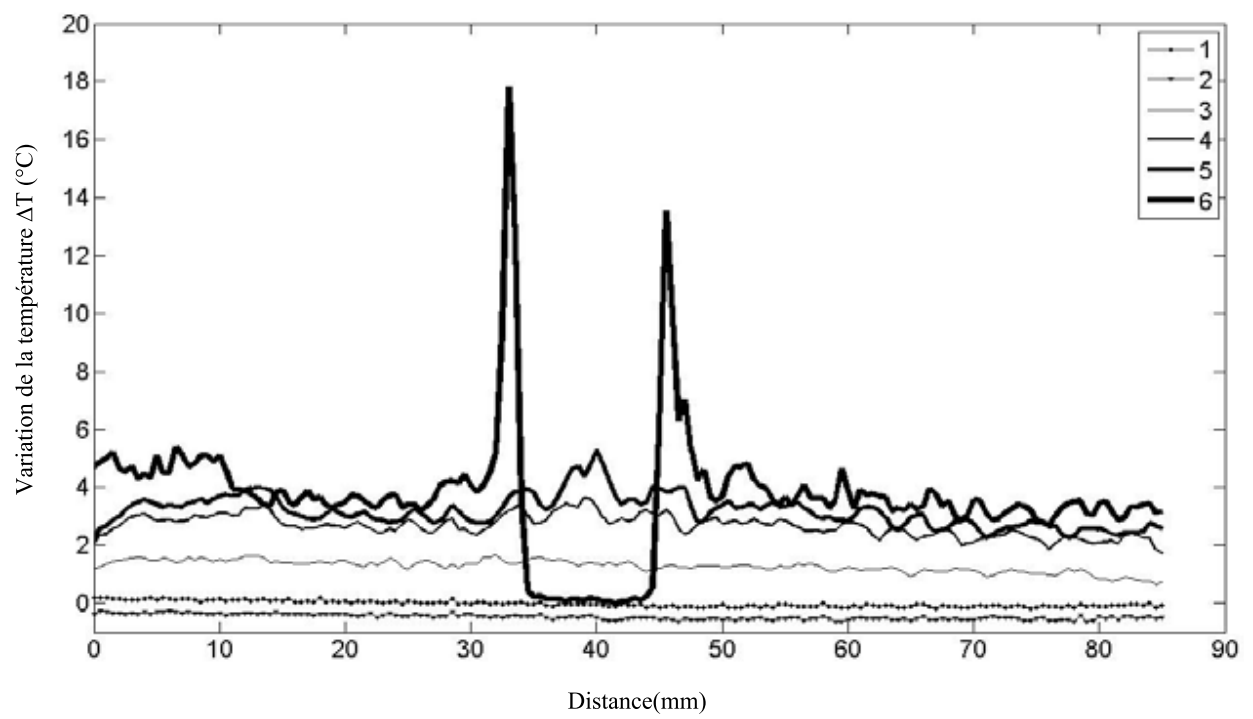

Fig. 8. Profils de température le long d'une ligne sur l'axe de l'éprouvette du PA10FV.

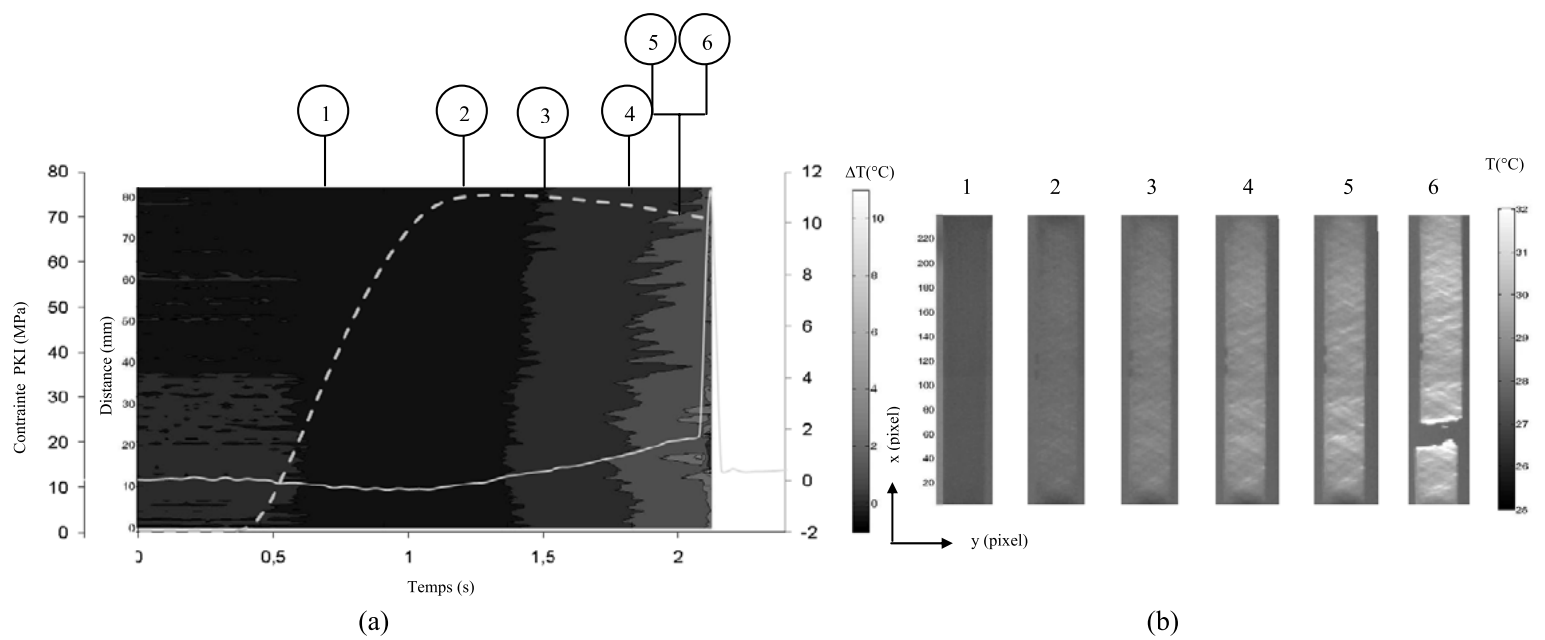

Fig. 9. (a) Courbe 1 : évolution temporelle des profils longitudinaux-courbes 2 et 3 : évolution de la charge et de la température (en un point particulier); (b) photogrammes thermiques de la surface de l'éprouvette à des temps différents du PA20FV (1 pixel représente $0,5 \mathrm{~mm})$.

\subsubsection{Cas du polyamide non chargé}

Dans le cas du polyamide non chargé (PA00FV) : la courbe de traction se décompose schématiquement de trois stades successifs : un domaine quasi linéaire suivi d'un domaine non-linéaire correspondant au développement de déformations inélastiques où la contrainte évolue pour atteindre un «plateau plastique » correspondant à la propagation des lèvres de striction. La distribution du champ de température peut être décomposée de la même façon (Fig. 6a : évolution de la température en un point particulier). Dans une première étape, le couplage thermoélastique induit une diminution de la température. S'en suit, du fait de la dissipation associée au développement de la viscoplasticité au sein de l'éprouvette, une augmentation homogène du champ de température. On observe ensuite une forte augmentation de la température associée à un phénomène de localisation-striction. Comme il est classiquement observé dans le cas du PA00FV, la striction se propage au sein de l'éprouvette suivant deux fronts se déplaçant en sens inverse, clairement identifiables sur les profils de température (Fig. 6b). La rupture finale se situe dans la zone de forte localisation.

\subsubsection{Cas du polyamide chargé}

On observe dans un premier temps, dans la zone de comportement quasi-linéaire, une évolution homogène du champ de température. Cependant, à la différence du PA00FV, l'analyse du champ thermique des PA10FV, PA20FV et PA30FV indique une forte hétérogénéité thermique dans la zone de comportement non-linéaire. Les 


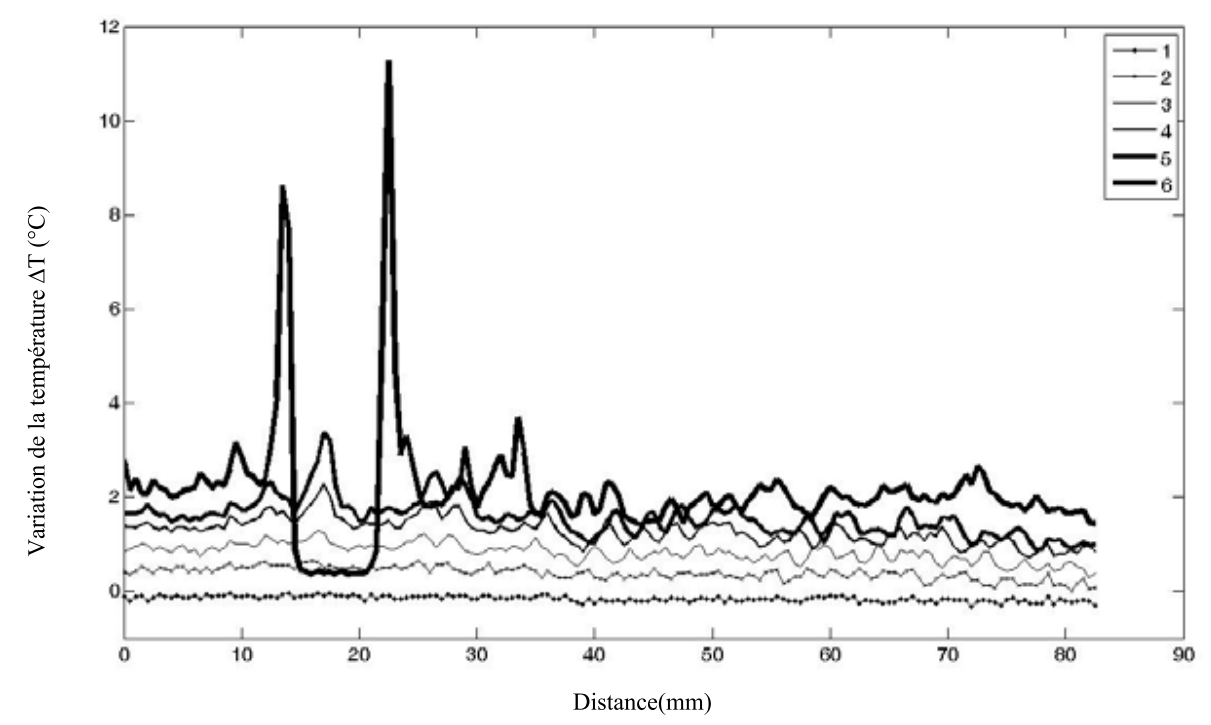

Fig. 10. Profils de température le long d'une ligne sur l'axe de l'éprouvette du PA20FV.

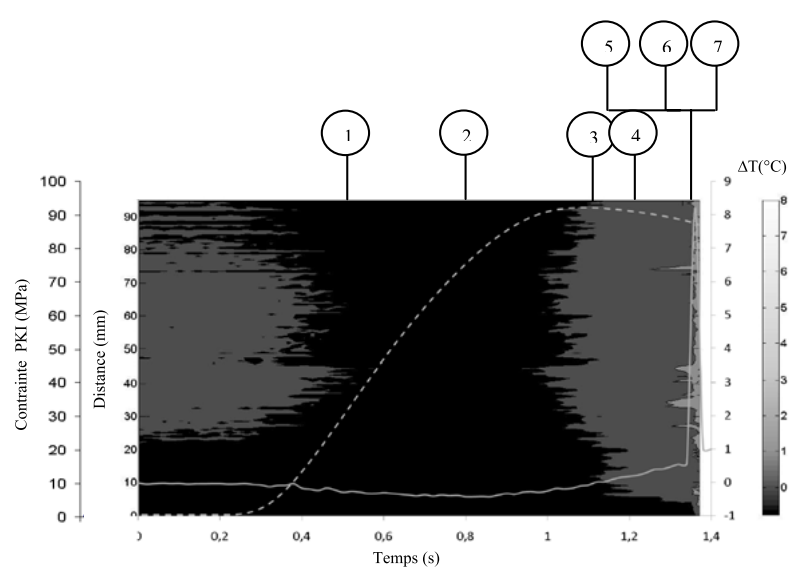

(a)

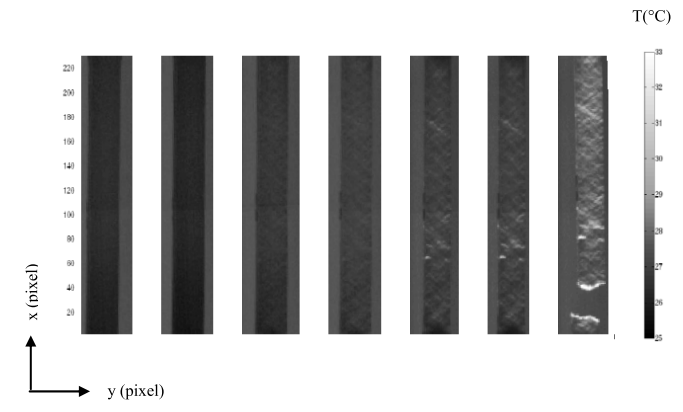

(b)

Fig. 11. (a) Courbe 1 : évolution temporelle des profils longitudinaux-courbes 2 et 3 : évolution de la charge et de la température (en un point particulier); (b) photogrammes thermiques de la surface de l'éprouvette à des temps différents du PA30FV (1 pixel représente $0,5 \mathrm{~mm})$.

sources de chaleurs sont dans un premier temps homogènes (Figs. 7a, 9a et 11a).

Cette observation est illustrée d'une autre manière par les courbes des profils longitudinaux extraites des images thermiques (Figs. 8 et 10). Dans une première étape, on observe un effet thermo-élastique homogène. Ce résultat est très similaire à celui obtenu dans le cas du matériau non chargé. On observe ensuite (étapes 3-6) une évolution de plus en plus hétérogène du champ thermique qui semble indiquer une localisation de plus en plus prononcée de la déformation. Il est également possible d'identifier des zones dont l'évolution de l'hétérogénéité du champ thermique est plus importante indiquant une seconde échelle d'hétérogénéité du champ thermique associé à la localisation progressive de la déformation. L'instant de la rupture est associé à une augmentation significative de la température sur l'ensemble de l'éprouvette.

Ces phénomènes d'hétérogénéités thermiques sont accentués en augmentant la vitesse de déformation et/ou de la quantité de fibres de verre courtes. La figure 12 représente l'évolution des profils le long d'une ligne sur l'axe de l'éprouvette dans le cas de trois valeurs de vitesses de déformations différentes du PA30FV.

Ces sources de chaleurs apparaissent sous forme de bandes thermiques et dissipatives (Figs. 7b, 9b et 11b) qui naissent puis se propagent dans des directions voisines de $45^{\circ}$. Ces bandes se trouvent également correspondre à des régions qui sont le siège d'importantes déformations qui se localisent à la surface (ou proche de la surface) de l'éprouvette. Les observations en microscopie optique et MEB confrontées avec les images thermiques (Figs. 13 et 14) indiquent que les zones de localisation thermique correspondent à des zones de déformations localisées importantes (blanchissement de la matière) voire des micro-fissurations généralement initiées à l'endroit où se croisent les zones de localisation (Fig. 14). Ces zones de localisation thermique sont associées aux bandes de cisaillement également identifiables sur les résultats 

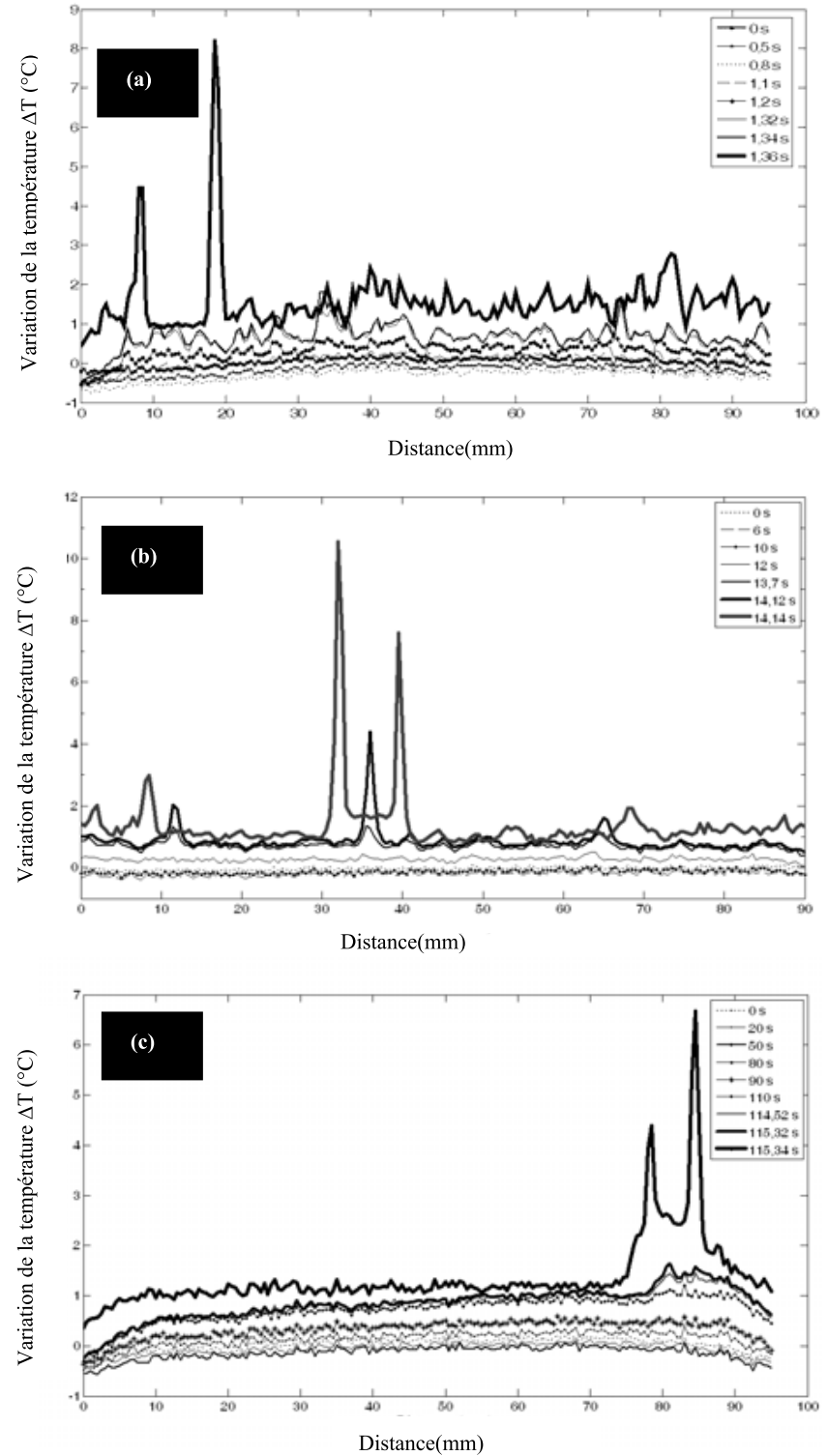

Fig. 12. Profils de température le long d'une ligne sur l'axe des éprouvettes PA30FV à (a) $\varepsilon=5,5 \times 10^{-2} \mathrm{~s}^{-1}$, (b) $\varepsilon^{\prime}=$ $5,5 \times 10^{-3} \mathrm{~s}^{-1}$ et $(\mathrm{c}) \dot{\varepsilon}=5,5 \times 10^{-4} \mathrm{~s}^{-1}$.

de corrélation d'images (Fig. 15). L'association thermographie infrarouge/DIC permet d'en déduire que les élévations de température observées sont les processus de déformation associés aux bandes de cisaillement qui se développent sur la surface de l'éprouvette. Deux échelles d'hétérogénéité apparaissent à la fois dans les images infrarouge et DIC. La première est associée à la présence des bandes de localisation, la deuxième à une densification de cette localisation révélant une interaction à plus grande distance entre ces zones de localisation. C'est cette seconde échelle qui aboutit au déclenchement de la fissuration et de la rupture finale de l'éprouvette. Les processus de déformation de type bande de cisaillement et l'évolution de leur hétérogénéité spatiale semblent dominer et contrôler le comportement à rupture des matériaux chargés.
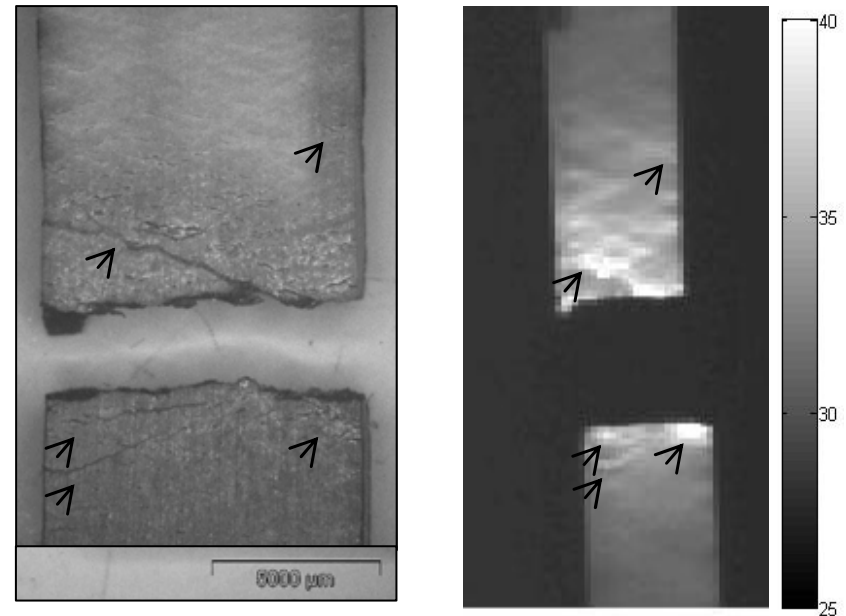

Fig. 13. Analyse du même échantillon PA10FV par : (a) microcopie optique binoculaire et (b) thermographie infrarouge.

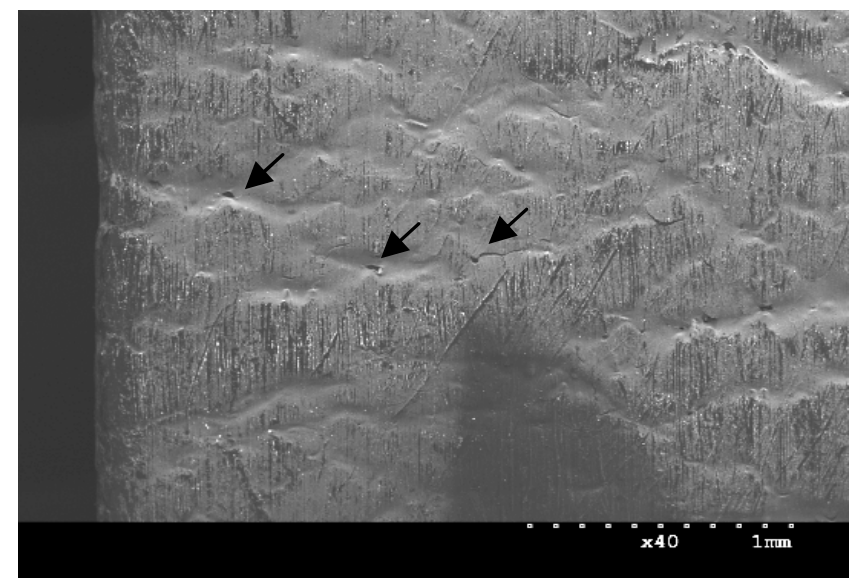

Fig. 14. Observations MEB de la surface des éprouvettes montrant des microfissurations de la matrice (PA10FV).

La figure 16 illustre une image 3D prise par microtomographie aux rayons $\mathrm{X}$ dans une zone correspondant aux zones associées aux fortes augmentations de la température juste avant rupture. On y observe clairement le développement d'une zone de fissuration importante en sous surface.

\subsection{Caractérisation microstructurale}

L'observation des faciès de rupture a été effectuée sur les éprouvettes testées à une vitesse de déformation et à un taux d'humidité constants $(\mathrm{RH}=50)$ afin d'étudier l'évolution des mécanismes d'endommagement en fonction du taux de fibres dans la matrice polymère. La figure 17 représente le faciès de rupture du polymère non chargé. Elle indique la présence de trois zones d'endommagement différentes : une première zone (zone 1) de croissance d'une cavité au cours de la déformation plastique (zone d'amorçage de la rupture), une deuxième zone 

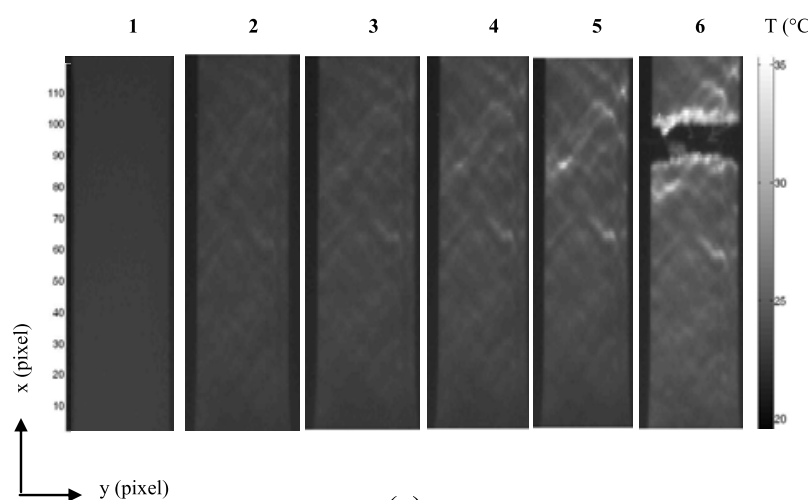

(a)
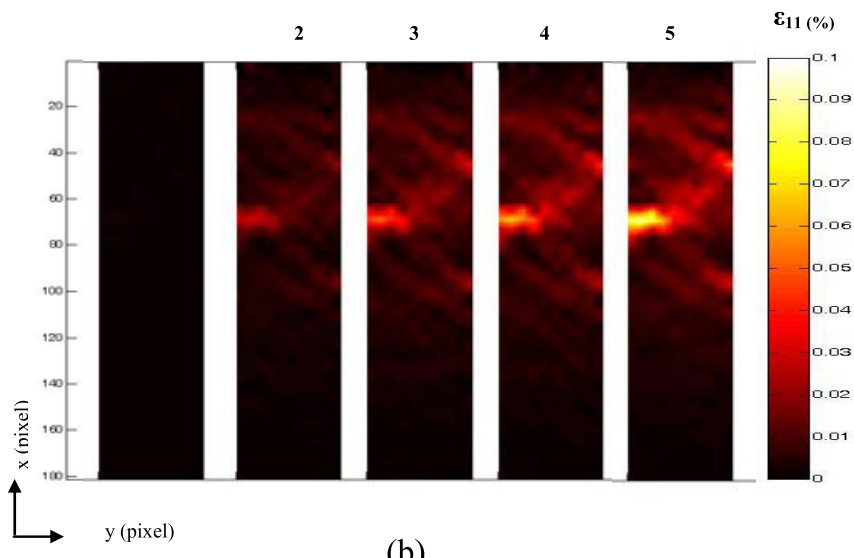

(b)

Fig. 15. (a) Photogrammes thermiques de la surface de l'éprouvette à des temps différents du PA20FV (1 pixel représente $0,2 \mathrm{~mm}$ ) ; (b) champ de déformation $\varepsilon_{11}$ à des temps différents du même matériau.

(zone 2) de propagation de la rupture suivant un plan perpendiculaire à la direction du chargement et finalement une troisième zone (zone 3 ) de rupture finale.

Ce résultat est en bon accord avec les résultats thermiques. En effet, la localisation très importante observée au centre de l'éprouvette (voir Fig. 6b : image 4 et Fig. 17 : zone 2) s'effectue de manière ductile et est due aux phénomènes d'apparition et de propagation de la striction qui mènent à la formation des fibrilles (Fig. 18). Ceci explique l'élévation plus importante de la température du polymère non chargé par rapport à celui chargé par fibres de verre.

Les processus mis en jeux dans le cas des polymères chargés sont très différents de ceux du polyamide non chargé (voir Fig. 19 dans cas du PA10FV). Le matériau présente trois zones de rupture :

- une zone d'amorçage de la rupture (zone 1), similaire à celle observée dans le cas du polymère non chargé mais de taille beaucoup moins importante. Cette zone semble également correspondre à un taux de fibres localement faible;

- le développement de l'endommagement se fait de manière ductile. On peut observer de très fortes déformations de la matrice dans les espaces interfibre (zone de déformation plastique à l'échelle

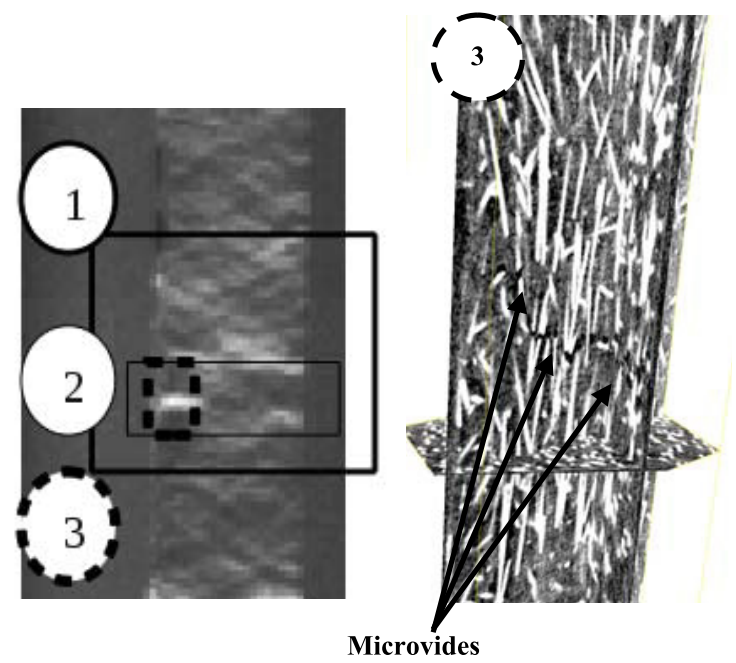

Fig. 16. Image 3D prise d'un volume de $1400 \times 400 \times 200 \mu \mathrm{m}^{3}$ d'un PA30FV (précision d'acquisition 1,2 $\mu \mathrm{m} /$ pixel).

microscopique: zone 2). La plupart des fibres sont orientées dans la même direction, certaines en faible quantité se trouvent dans le plan de rupture;

- la zone finale de rupture est de type quasi-fragile (zone 3) : la matrice ne montre quasiment pas de traces de déformations plastiques (la surface de rupture de la matrice présente une rugosité faible). La présence de cavités indique que les fibres ont été déchaussées au niveau de l'interface (phénomène de «pull-out $»)$.

La comparaison de l'image MEB avec celle thermique (Fig. 19) montre bien que l'amorçage de la fissure correspond aux zones ductiles tandis que sa propagation à travers l'éprouvette s'effectue d'une manière fragile et menant à la rupture finale. Les mêmes résultats ont été observés dans les matériaux chargés à 20 et $30 \%$ de fibres de verre. La principale différence est la transition en zone de rupture de type 3 beaucoup plus rapide avec l'augmentation du taux de fibres.

Les observations précédentes faites dans le cas du taux d'humidité $\mathrm{RH}=50$ sont similaires pour des taux d'humidité supérieurs. D'autre part, la matrice polymère présente une ductilité de plus en plus importante en fonction de l'humidité. Dans le cas de $\mathrm{RH}=70$, la matrice se détache complètement des fibres et s'étire de façon visqueuse en des ligaments étirés.

Sato et al. [11,12] décrivent les mécanismes de rupture des thermoplastiques à partir d'études faites sur le PA66 renforcé de fibres de verre courtes. Dans une approche basée sur l'émission acoustique et des observations microscopiques, les auteurs montrent que l'initiation de fissure se fait aux extrémités des fibres. Les microfissures se propagent ensuite le long de l'interface, sous l'effet de contraintes de cisaillement. Les endommagements créés, génèrent une bande de déformation plastique et mènent la matrice à sa déformation maximale. La bande plastique finit par engendrer une macrofissure, qui se propage 

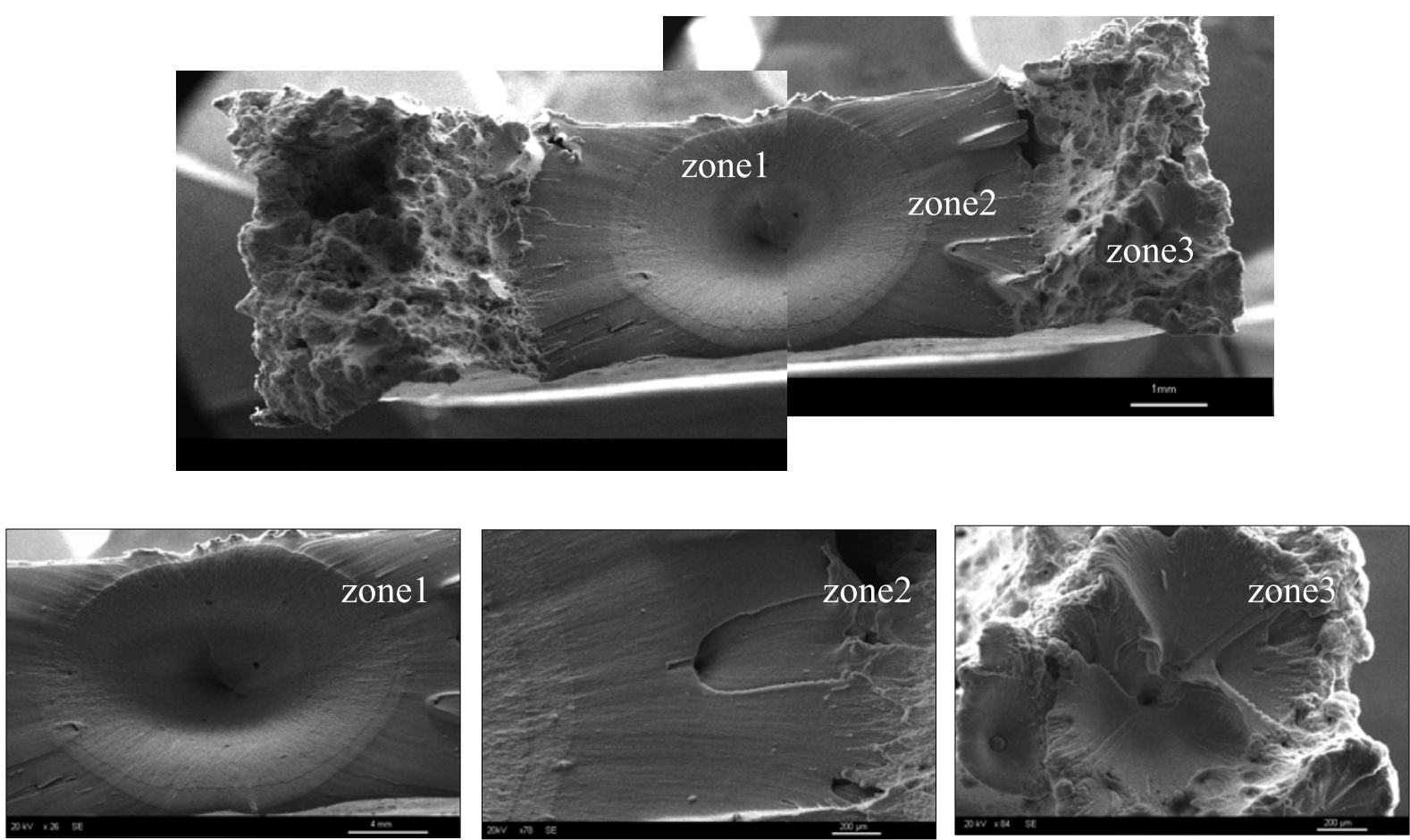

Fig. 17. Micrographies MEB du faciès de rupture du PA00FV à $\dot{\varepsilon}=5,5 \times 10^{-4} \mathrm{~s}^{-1}$ et $\mathrm{RH}=50$ montrant les trois zones d'endommagement.

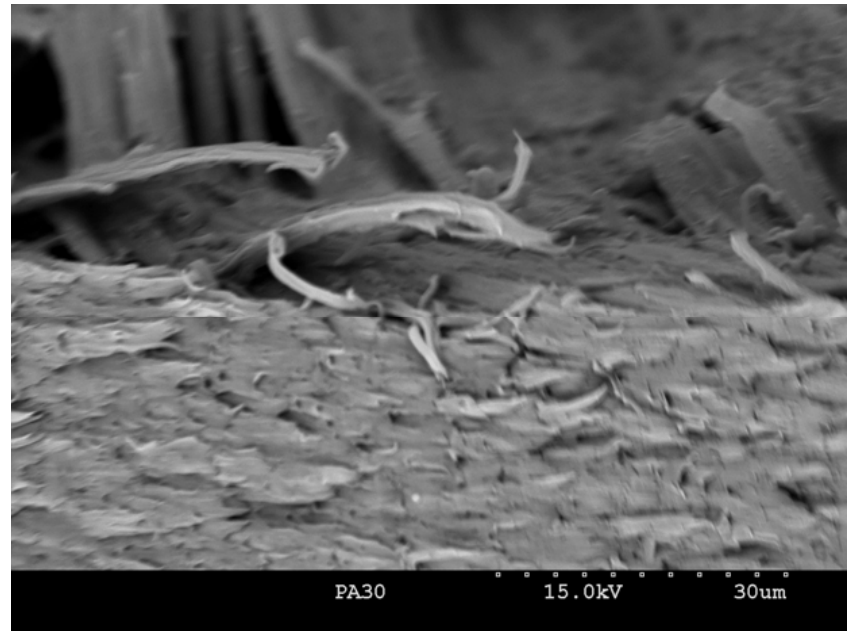

Fig. 18. Micrographies MEB du PA00FV montrant la formation des fibrilles.

lentement en déchaussant les fibres. Lorsque la fissure atteint sa taille critique, une rupture brutale se produit.

Dans son étude sur le polypropylène renforcé par des fibres de verre, Sova [13] propose une description schématique pour illustrer l'endommagement de ce matériau. Il explique que dans le cas d'une faible adhérence entre les fibres et la matrice, la ruine du matériau est due au cisaillement de la matrice et à sa déformation plastique.

L'analyse des résultats, obtenus par les différentes investigations, permet de corréler la localisation thermique avec la microstructure du matériau et de mettre en évidence les similitudes des phénomènes observables et établir des analogies : dans le matériau composite, soumis à une traction uniaxiale, la matrice polyamide subit des déformations impliquant des lamelles cristallines séparées par des zones interlamellaires amorphes. Sous l'effet de la contrainte, ces lamelles subissent un glissement les unes par rapport aux autres créant ainsi des bandes de cisaillement. Ces bandes constituent un mécanisme élémentaire de déformation systématiquement associée à une élévation de température localisée. Leurs multiplications et leurs propagations en zones intenses sont les précurseurs à la rupture finale. Cette dernière phase d'endommagement est marquée par un saut rapide de la température. Elle est liée au couplage thermo-élastique et à la présence de phénomènes dissipatifs type frottement fibre-matrice au niveau de l'interface et directement lié au phénomène de décohésion progressive fibre-matrice se développant au cours de l'essai de traction.

\section{Conclusion}

Dans cette étude l'effet du taux de fibres de verre, vitesse de déformation et du taux d'hygrométrie a été étudié. La distribution surfacique des sources de chaleur durant le processus de déformation en traction a été obtenue à l'aide de la technique de thermographie infrarouge. Sur les différentes éprouvettes nous avons pu observer les phénomènes dissipatifs de localisation. Ces phénomènes sont différents dans le cas du polymère non chargé et chargé de fibres de verre. 

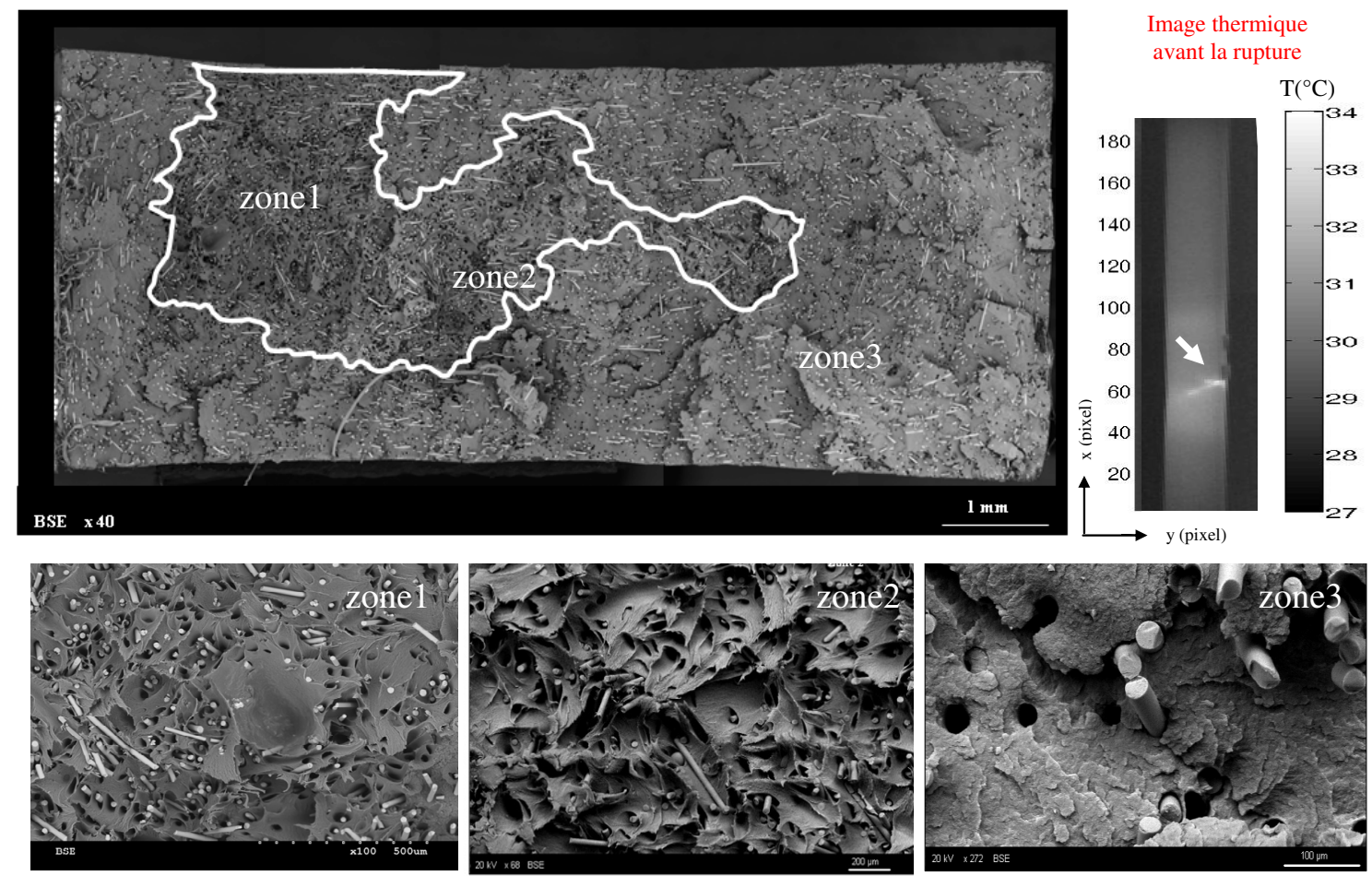

Fig. 19. Micrographies MEB du faciès de rupture du PA10FV à $\dot{\varepsilon}=5,5 \times 10^{-4} \mathrm{~s}^{-1}$ et $\mathrm{RH}=50$.

\section{Références}

[1] M.I. Cohan, Nylon plastics handbook, Hanser Gardner Publications, 1995

[2] J.P. Tancrez, J. Pabiot, F. Rietsch, Damage and fracture mechanisms in thermoplastic-matrix composites in relation to processing and structural-parameters, Compos. Sci. Technol. 56 (1996) 725-731

[3] W.N. Ota, S.C. Amico, K.G. Satyanarayana, Studies on the combined effect of injection temperature and fiber content on the properties of the polypropylene-glass fiber composites, Compos. Sci. Technol. 65 (2005) 873-881

[4] A. Gülü, A. Özdemir, E. Özdemir, Experimental investigation of the effect of glass fibres on the mechanical properties of polypropylene (PP) and polyamide 6 (PA6) plastics, Mater. Des. 27 (2006) 316-323

[5] B. Mouhmid, A. Imad, N. Benseddiq, S. Benmedakhène, A. Maazouz, A study of the mechanical behaviour of a glass fibre reinforced polyamide 6,6: Experimental investigation, Polymer. Tes. 25 (2006) 544-552

[6] Yuanxin Zhou, P.K. Mallick, A non-linear damage model for the tensile behaviour of an injection molded short Eglass fiber reinforced polyamide-6,6, Mat. Sci. Eng. A 393 (2005) 303-309
[7] J.L. Thomasson, The influence of fibre properties of the performance of glass-fibre-reinforced polyamide 66 , Compo. Sci. Technol. 59 (1999) 2315-2328

[8] D. Valentin, F. Paray, B. Guetta, The hygrothermal behaviour of glass fibre reinforced PA66 composites: a study of the effect of water absorption on their mechanical properties, J. Mater. Sci. 22 (1987) 46-56

[9] M. Akay, Moisture absorption and its influence on the tensile properties of glass fibre reinforced polyamide 6,6 , Polym. Polym. Compos. 2 (1994) 349-354

[10] J.L. Thomasson, Micromechanical parameters from macromechanical measurements on glass reinforced polyamide 6,6, Compo. Sci. Technol. 61 (2001) 2007-2016

[11] T. Sato, S. Kurachi, S. Sato, O. Kamigaito, Microfailure behaviour of randomly dispersed short fiber reinforced thermoplastic composites obtained by direct SEM Observation, J. Mater. Sci. 26 (1991) 3891-3898

[12] T. Sato, S. Kurachi, S. Sato, O. Kamigaito, Mechanism of fracture of short glass fibre-reinforced polyamide thermoplastic, J. Mater. Sci. 19 (1984) 1145-1152

[13] M. Sova, The morphology of fracture surface and mechanical properties of composite of polypropylene with glass fibres having different interface adhesion, J. Appl. Poly. Sci. 38 (1989) 511-528 